\title{
Item-presentation controls for multidimensional item pools in computerized adaptive testing
}

\author{
THOMAS J. THOMAS \\ Johns Hopkins University, Baltimore, Maryland
}

\begin{abstract}
Item pools or item banks used in most testing situations are inherently multidimensional. This is especially a problem in computerized adaptive testing (CAT), which is driven by item response theory; item response theory requires that the item pool be unidimensional. This series of computer simulations demonstrates how alternative item-presentation controls (content-balancing and "mini-CATs") may be employed in CAT to estimate ability accurately in spite of the violation of unidimensionality. Averaged, shorter mini-CATs provide the most accurate estimation of ability and ameliorate problems intrinsic to violating the unidimensionality assumption of item response theory.
\end{abstract}

The introduction of item response theory, a subclass of latent structure models, has changed the face of psychometrics and educational testing. Many specific areas that are benefiting greatly include the assessment of item bias (Ironson, 1983), test equating (Baker, 1984; Lord, 1980), estimation of ability parameters with increased accuracy of measurement at the typically difficult extreme ranges of ability populations (i.e., especially low or high abilities) (Lord, 1977), and computerized adaptive testing (or, tailored testing) (Green, 1970, 1983; Weiss, 1982). The latter application especially, coupled with the speed of the computer, has opened up new frontiers hitherto unexplorable by conventional means.

Studied under different names since the 1940s (viz., latent trait test theories; item characteristic curve theory), item response theory stipulates a specific item response model in the form of a conditional probability function, $P_{i}(\theta)=P\left(u_{i}=1 \mid \theta\right)$, where $P_{i}(\theta)$ defines the probability of a correct response to item $i$. The graph of the function, called an item characteristic curve, represents the regression of observed scores (across a population of examinees) for a single item on a single underlying trait. Several models-mostly for unidimensional latent traitshave been differentiated on the basis of the number of unknown parameters to be estimated. The complete model to be discussed here is the three-parameter logistic model (Birnbaum, 1968, p. 405), which states:

$$
P_{i}\left(\theta_{a}\right)=\frac{c_{i}+\left(1-c_{i}\right)}{\left\{1+\exp \left[-1.7 a_{i}\left(\theta_{a}-b_{i}\right)\right]\right\}}
$$

where $P_{i}\left(\theta_{a}\right)$ is the probability of an examinee's answering item $i$ correctly; $c_{i}$ is the lower asymptote for item

\footnotetext{
The author would like to thank his advisor, Bert F. Green, for his tireless assistance and advice, ceaseless wit, and patience with programming that did not always do what we wanted it to but always did what we told it to do. Correspondence may be addressed to Thomas J. Thomas, Department of Psychology, The Johns Hopkins University, 34th and Charles Streets, Baltimore, MD 21218.
}

$i$ (sometimes called a pseudo-chance-level or guessing parameter); $a_{i}$ is the discrimination parameter for item $i ; \theta_{a}$ is the ability of examinee $a$; and $b_{i}$ is the difficulty index of item $i$. According to this model, an examinee has some calculable probability of correctly answering an item as a function of his or her own ability and the characteristics of the item itself.

These characteristics of the item, the item parameters, have their parallel in classical test theory. The item discrimination parameter, $a$, is more precisely a measure of the slope of the regression of observed scores on ability level at the inflection point of that curve. The slope, then, represents the nature of the item's discrimination of ability $(\theta)$. A flat slope would suggest that the item does a poor job discriminating ability, whereas a steep slope would indicate that the item discriminates well-but over a narrow range of ability. Note that the preferred steeper slope (indicating good discrimination) has as its drawback the fact that the item trades high discrimination for a narrow range of that high discrimination.

The item difficulty parameter $b$ also has a parallel parameter in classical test theory. A measure of the inflection point on the regression curve that intersects the abscissa (the ability $[\theta]$ axis), $b$ can be graphically represented as the degree to which the entire item characteristic curve is shifted to the left or right relative to the ability scale of interest. A larger value of $b$ for a particular item indicates a concomitant increase in ability required to successfully answer the item correctly.

The guessing parameter $c$ is not easy to estimate, because its behavior is not well understood. This parameter can be graphically understood as the lower asymptote of the item characteristic curve. One- and two-parameter models do not concern themselves with the estimation of this parameter, but its effects cannot be disregarded without some concern as to how the "real-world" likelihood of guessing affects the mathematical relationships between latent constructs and measured variables. Because this guessing parameter has proven difficult to estimate 
with any consistency or stability, it is often set at a specified, but reasonable, value based on the number of alternative answers for any specific item.

Whereas item response theory has always had the potential to increase the statistical repertoire of the psychometrician, the practical limitations imposed by the amount and complicated nature of the numerical manipulations involved have operationally precluded the psychometrician from utilizing item response theory as a regular tool. The introduction of the computer into the laboratory as a common-if not necessary-tool, and its accompanying ability to make multiple calculations, store vast amounts of data, and manipulate incoming information in the form of physical responses at the computer keyboard, has provided the psychometrician with a powerful ally. By combining the computer's speed of calculation with the developing field of tailored testing and item response theory, the advent of computerized adaptive testing was made possible.

The full acceptance and implementation of computerized adaptive testing faces several hurdles, the presence of multidimensionality in the item pool being one such formidable problem. However, it may be possible to arrive at accurate estimates of ability $(\theta)$-employing item response theory as our underlying model-in spite of this multidimensionality.

\section{The Assumption of Unidimensionality}

The mathematical model underlying item response theory is founded on several very strong assumptions, the most important one being the assumption that the items in the population of interest are unidimensional. There are several areas in which violation of this assumption may be made manifest: (1) during estimation of item parameters, and (2) during estimation of ability estimates. Ansley and Forsyth (1985) have studied the effects of dimensionality on parameter estimations by systematically changing the correlations between two underlying ability dimensions. They discovered that the resultant estimated parameters, for the most part, could be interpreted as averages of the parameters from the two underlying dimensions. Greaud (1987) investigated the effects of violation of the unidimensionality assumption on parameter estimation and item selection, and extended it to the estimation of ability in the context of computerized adaptive testing (CAT). She examined both between-item multidimensionality and within-item multidimensionality. ${ }^{1}$ She found that, in groups of examinees whose ability estimates for two separate but related dimensions were correlated only slightly, multidimensional between-item structure in the item pool tended to yield item discrimination parameters that estimated one of the dimensions on that parameter better than the other dimension. She concluded that the iterative process in the estimation procedures was culpable and inescapable, and that it would most likely lead to an imbalance in the construction of an adaptive test.

\section{Item-Selection Controls}

There are at least two possible avenues for handling a multidimensional item pool with a model that requires the items be unidimensional: We can simply ignore the violation of unidimensionality and administer the CAT, hoping that the theta estimates accurately measure the underlying abilities; or we can place some sort of item-selection control in the test to overcome the violation. Plausible item-selection controls are: (1) "content-balancing, " an algorithm in the CAT procedure that forces the program to alternate sampling 10 items from each of the two subsets making up the one large pool of items, where each subset is a homogeneous collection of items that determine one of the two underlying dimensions, or (2) dividing the CAT into two separate 10-item tests ("mini-CATs") on the basis of "dimension-ness" of the item pools. The ability estimates could be calculated via two CAT administrations on the separate dimensions, then averaged over the two CATs as a measure of overall ability.

Of secondary interest is whether shorter, five-item CATs ("micro-CATs") would yield reasonably accurate ability estimates, useful perhaps, for a "quick and dirty" estimate of ability. This latter question would also yield information about the speed (rate) at which the $\theta$ centers on its final estimate.

This series of computer simulations was designed to investigate the effect of implementing these two itemselection controls in simulated CATs of various lengths where item pools had been defined-or set up-to be multidimensional. The results of the item-selection controls were compared to a situation in which a CAT had been administered in the usual fashion, ignoring the fact that the item pool was not unidimensional (as required by the underlying item response theory model). The entire process was replicated with a second set of generated items and population parameters.

To investigate the consistency of ability estimation across the two-dimensional theta space that defines all of the possible combinations of two ability levels, the procedures were repeated for nine different pairs of theta (ability) coordinates.

\section{Hypotheses}

Greaud (1987) examined problems with item parameter estimation techniques when dealing with two-dimensional between-item test items. On the basis of her results, it can be hypothesized that almost any item-selection algorithm implemented in a CAT with multidimensional items will be better and will result in more accurate estimations of ability $(\theta)$ than no control will. Hence, both content-balancing and the smaller mini-CATs should result in better estimates of overall ability than simply ignoring the multidimensional nature of the item pool and performing a single CAT. As the correlations of the underlying abilities approach unity, however, the advantage of both item-selection procedures should become less 
pronounced or disappear entirely, because a larger correlation between underlying abilities will make the items appear more and more unidimensional.

The real hope, however, lies with the content-balancing estimation procedure; if the procedure behaves correctly, ability estimates by content-balancing should be more accurate across all variations of between-item correlation for both the single CAT and the averaged mini-CATs.

It was predicted that a similar pattern of results would exist for the five-item micro-CATs, with the exception that the magnitude of all effects would be diminished.

\section{METHOD}

\section{Data Generation Procedures}

A customized CAT program provided the backdrop for conducting all of the following manipulations on item pools and estimation controls.

Simulated item parameters and examinee populations were generated and compared to estimated item and ability parameters. The power in this approach is that the true nature of the parameters is "known"-they have been generated ahead of time and constrained (according to rules) to simulate real populations of test items and examinees. The estimated parameters (both item and ability) can be compared against these "true" values to calculate accuracy and stability of the estimates.

Simulated examinees. Each examinee was assigned one ability on each of two dimensions where each ability is normally distributed. The abilities on the two dimensions are assumed to be correlated across examinees, to a certain extent. In this study, three values of the correlation were used, thereby effectively defining three different populations; these correlations were $0.4,0.6$, and 0.8 . For each of the three populations, 2,006 simulated cases were sampled.

Simulated items. In a two-dimensional world, each item necessarily has two discrimination parameters, $a_{1}$ and $a_{2}$; it was assumed that each item had one zero $a$, on one or the other dimension. Totals of 50 items of each type were used.

Item parameter selection. To obtain item parameters for 100 items, 100 sets of "true" item parameters $(a, b$, and $c$ ) conforming to the three-parameter logistic model were first generated, subject to the constraints that: (1) The nonzero item discrimination parameter $(a)$ was uniformly distributed in the interval between 1.2 and 1.8, (2) the item difficulty parameter $(b)$ was triangularly distributed in the interval between values of -2.0 and 2.0 , and (3) the guessing parameter $(c)$ was set at 0.2 .

A set of item response data was then generated from each of the three examinee populations. Item parameters for a one-dimensional item response theory model were estimated for each set; the same model was also fit to the 50-item unidimensional subsets for each examinee population. Both $a$ s and $b$ s were estimated according to Lord's (1980) maximum likelihood procedure, while Bayes's modal estimation was used to calculate the ability esti- mates $(\theta)$. This procedure is a hybrid of the Bayesian methods of Swaminathan and Gifford (1986), and it is the more common method used by the LOGIST computer program (Wingersky, 1983). This combination of techniques was meant to lend consistency to the program of estimation, since a Bayesian modal estimator was used in the CAT simulations.

\section{Simulated CATs and Related Controls}

Three different modes of CAT were run on every combination of correlated ability levels. The first CAT was simply a simulation of a 20 -item CAT, ignoring the known dimensionality of the test items. The second CAT performed the function of content-balancing. In addition to calculating ability estimates according to the normal routine in CAT-estimating ability following the administration of each item and selecting the next item subject to the rule that it provide maximum information for that theta level-this routine forced the estimation process to sample alternately from items in two separate item pools. Each item pool contained items that were unidimensional to that item pool but multidimensional across item pools. This alternating sampling continued until the requisite 20 items had been "taken," thereby terminating the test. Both the standard CAT and the content-balanced CAT used the estimated item parameters for the 100 -item pools. The third and final CAT was really two CATs. The 100 -item pool was split into two 50-item pools, and two 10 -item CATs were simulated, one on each item pool, using the item parameters from the estimation for the 50 -item subsets. The ability estimates from each simulation were averaged to yield a single score for ability. It should be noted that, in all cases, the actual item responses were simulated from the "true" two-dimensional item parameters.

A secondary set of simulations entailed simulating 5item micro-CATs and comparing the direction and magnitude of these results to that of the longer 10-item mini-CATs.

\section{Analyses}

All of the estimated ability scores $(\theta)$ derived from the various procedures (normal CAT, content-balanced CAT, averaged mini-CATs) across all of the manipulated correlations of underlying ability or pairs of ability scores $(0.4$, $0.6,0.8$ ) were correlated with the known or "true" ability scores. Because two separate known dimensions of ability were defined and correlated to some degree, it is reasonable to also define a target true theta, which would be calculated as the simple arithmetic average of the two underlying ability $\theta \mathrm{s}$. To obtain "accuracy" measures for the item-selection procedures, the magnitudes of the discrepancies between estimated and known ability parameters were inspected.

\section{Replication}

Finally, the entire process was replicated with a new set of known item parameters and population ability parameters to measure the stability of the item-selection 
controls across new item pools and populations. The same interdimension correlations were maintained for this replication, and identical analyses were performed.

\section{RESULTS AND DISCUSSION}

The mean item parameters and standard deviations for the original 100 items, as well as the shapes of the respective distributions, were examined and found to be well behaved. Inspection of these summary statistics for all of the conditions reveals that the item parameters did indeed conform nicely to the desired constraints imposed.

The correlations of the true thetas-and an average of the true thetas (target theta)-with the estimated thetas for the three modes of item selection and for interdimension correlations of $0.4,0.6$, and 0.8 are displayed in Table 1 . Content-balancing the item selection for underlying dimensions resulted in a higher correlation of the estimated theta with the known true theta than a regularly administered CAT when item pools were correlated both 0.4 and 0.6 . The effect was absent when the pools were correlated 0.8; that is, when the item pools appeared (psychometrically) to be unidimensional in makeup, it made little, if any, difference to content balance. Greaud (1987) demonstrated the "emphasizing" nature of the estimation program-in a regularly administered CAT - when the items actually represent two different dimensions. She pointed out that the program would unfairly emphasize one of the dimensions at the expense of the other; the selected items would have represented one of the underlying dimensions to a greater degree than the other, the choice of the dimension a completely arbitrary one. The content-balancing procedure minimized the effect of the "emphasizing," but at the cost of lowering the correlation between estimated and true theta of the previously emphasized dimension. Note that averaging two separate 10-item mini-CATs for each underlying dimension yielded the strongest correiations between estimated and true theta for each dimension as well as for an average theta across both dimensions. What we may conclude here is that, although content-balancing does indeed offer some

Table 1

Correlation Coefficients: True Thetas with Estimated Thetas

\begin{tabular}{lccc}
\hline & \multicolumn{2}{c}{ Dimension } & \\
\cline { 2 - 3 } Test Mode & Theta 1 & Theta 2 & Average \\
\hline & $r=0.4$ & & \\
Regular & .50 & .93 & .85 \\
Content-balanced & .68 & .87 & .91 \\
Averaged mini-CATs & .80 & .80 & .95 \\
& $r=0.6$ & & \\
Regular & .69 & .93 & .90 \\
Content-balanced & .83 & .87 & .95 \\
Averaged mini-CATs & .86 & .85 & .95 \\
& $r=0.8$ & & \\
Regular & .90 & .91 & .95 \\
Content-balanced & .92 & .91 & .96 \\
Averaged mini-CATs & .92 & .91 & .96 \\
\hline
\end{tabular}

Table 2

Correlation Coefficients: True Thetas with Estimated Thetas (Replication)

\begin{tabular}{lccc}
\hline \multirow{1}{*}{ Test Mode } & \multicolumn{2}{c}{ Dimension } & \\
\cline { 2 - 3 } & Theta 1 & Theta 2 & Average \\
\hline & $r=0.4$ & & \\
Regular & .92 & .52 & .85 \\
Content-balanced & .52 & .71 & .92 \\
Averaged mini-CATs & .80 & .81 & .95 \\
& $r=0.6$ & & \\
Regular & .92 & .71 & .90 \\
Content-balanced & .87 & .83 & .94 \\
Averaged mini-CATs & .85 & .86 & .95 \\
& $r=0.8$ & & \\
Regular & .92 & .90 & .95 \\
Content-balanced & .91 & .96 & .96 \\
Averaged mini-CATs & .91 & .91 & .96 \\
\hline
\end{tabular}

amelioration of a poor estimation of ability when item pools are multidimensional (or at least bidimensional), the preferred method may be to administer smaller CATs and then average results to yield the most accurate estimation of the subject's "overall" underlying true ability across several dimensions.

Table 2 shows the results from the replication of the study with new bidimensional item pools and new item parameters. Strikingly similar correlations resulted between estimated and true thetas for all dimensions and all modes of CAT administration when comparison was made with the first study.

It appears, then, that the estimation and averaging procedures are consistent and stable. What is noteworthy in the replication is the arbitrary nature of the estimation program to "choose" one of the dimensions to emphasize for the CAT simulation. In the former simulation, Dimension 2 was emphasized, whereas in the latter simulation, more items representing Dimension 1 were selected in the simulation, yielding an artificial accent on that dimension.

\section{STABILITY OF ESTIMATION ACROSS TWO-DIMENSIONAL THETA SPACE}

An additional question dealt with the stability of our three CAT mode administration procedures at all three interdimension correlations. It is important to assert that the calculations are performed with the same degree of accuracy and consistency both across modes of administration (regular, content-balanced, and averaged 10-item CATs), and across the two-dimensional theta space that defines the possible combinations of an examinee's ability level on two dimensions.

To investigate this consistency, nine pairs of ability coordinates were "fixed" in two-dimensional theta space. These pairs of coordinates represented pairs of possible theta values on two item pool dimensions; each theta corresponds to its respective value on an ability level or dimension. CATs were simulated for 100 ability-identical 
simulated examinees in each administrative mode (regular, content-balanced, or 10-item averaged). Inspection of the standard deviations and root mean square errors (see Tables 3 and 4 , respectively) of the resulting estimated thetas at each pair of true theta coordinates revealed a narrow range of errors both within interdimensional correlation and across administration mode. The typical standard deviation hovered around .29 , and the root mean square errors revealed errors that ranged between .24 and .39. The narrow band of the error estimates and the magnitude of the errors suggest that the procedures are, indeed, consistent.

\section{Five-Item "Micro-CATS"}

As before, a regular CAT and a content-balanced CAT - both with 10 items only-and two averaged 5-item CATs were all simulated on a new bidimensional item pool of 100 items, and the correlations of the estimated thetas with the true thetas were calculated. It should be

Table 3

Standard Deviations of Theta Estimates in a Two-Dimensional Theta Space

\begin{tabular}{lccccccccc}
\hline & \multicolumn{7}{c}{ Theta Coordinates } \\
\cline { 2 - 10 } Dimension 1 & .3 & .5 & .7 & -.2 & 0 & .2 & -.7 & -.5 & -.3 \\
Dimension 2 & .7 & .5 & .3 & .2 & 0 & -.2 & -.3 & $-.5-.7$ \\
\hline & \multicolumn{7}{c}{$r=0.4$} \\
Regular & .29 & .27 & .26 & .29 & .23 & .26 & .27 & .29 & .28 \\
Content-balanced & .31 & .31 & .32 & .39 & .29 & .29 & .33 & .30 & .30 \\
Mini-CATs & .26 & .24 & .23 & .26 & .28 & .25 & .32 & .29 & .27 \\
& & & $r=0.6$ & & & & & \\
Regular & .32 & .29 & .33 & .31 & .27 & .22 & .33 & .31 & .27 \\
Content-balanced & .26 & .33 & .34 & .25 & .26 & .30 & .32 & .28 & .27 \\
Mini-CATs & .30 & .27 & .26 & .26 & .29 & .24 & .29 & .24 & .33 \\
& & & $r=0.8$ & & & & & \\
Regular & .23 & .27 & .29 & .37 & .24 & .22 & .39 & .30 & .28 \\
Content-balanced & .29 & .31 & .32 & .33 & .29 & .26 & .30 & .28 & .31 \\
Mini-CATs & .29 & .29 & .29 & .29 & .29 & .28 & .27 & .26 & .30 \\
\hline
\end{tabular}

Table 4

Root Mean Square Errors of Theta Estimates in a Two-Dimensional Theta Space

Theta Coordinates

\begin{tabular}{|c|c|c|c|c|c|c|c|c|}
\hline \multirow[b]{2}{*}{ Dimension 1} & \\
\hline & .3 & .5 & .7 & -.2 & 0 & .2 & -.7 & $-.5-$ \\
\hline Dimensior & .7 & .5 & .3 & .2 & 0 & -.2 & -.3 & $-.5-.7$ \\
\hline \multicolumn{9}{|c|}{$r=0.4$} \\
\hline Regular & .33 & .28 & .33 & .37 & .25 & .28 & .37 & .33 .28 \\
\hline Content-balanced & .32 & .30 & .33 & .40 & .30 & .30 & .36 & .32 .32 \\
\hline Mini-CATs & .27 & .27 & .25 & .27 & .28 & .25 & .35 & .35 .32 \\
\hline \multicolumn{9}{|c|}{$r=0.6$} \\
\hline Regular & .37 & .30 & .39 & .36 & .27 & .28 & .39 & .32 .27 \\
\hline Content-balanced & .27 & .35 & .35 & .27 & .27 & .30 & .35 & .30 .28 \\
\hline Mini-CATs & .30 & .28 & .28 & .25 & .30 & .27 & .33 & .30 .36 \\
\hline \multicolumn{9}{|c|}{$r=0.8$} \\
\hline Regular & .25 & .28 & .32 & .37 & .25 & .22 & .39 & .30 .30 \\
\hline Content-balan & .30 & .32 & .32 & .33 & .28 & .27 & .30 & $.28 \quad .32$ \\
\hline Mini-CATs & .27 & .30 & .30 & .32 & .28 & .32 & .32 & $.32 \quad .35$ \\
\hline
\end{tabular}

Table 5

Correlation Coefficients: True Thetas with Estimate Thetas (Micro-CATs)

\begin{tabular}{lccc}
\hline \multirow{2}{*}{ Test Mode } & \multicolumn{2}{c}{ Dimension } & \\
\cline { 2 - 3 } & Theta 1 & Theta 2 & Average \\
\hline & $r=0.4$ & & \\
Regular & .41 & .92 & .79 \\
Content-balanced & .65 & .82 & .87 \\
Averaged mini-CATs & .75 & .74 & .88 \\
& $r=0.6$ & & \\
Regular & .64 & .91 & .86 \\
Content-balanced & .79 & .83 & .90 \\
Averaged mini-CATs & .82 & .81 & .90 \\
& $r=0.8$ & & \\
Regular & .85 & .88 & .91 \\
Content-balanced & .88 & .88 & .92 \\
Averaged mini-CATs & .87 & .87 & .91 \\
\hline
\end{tabular}

noted that this particular choice of length of CATs is not arbitrary. The interest was in the performance of the averaged 5-item CATS, a reasonable worst case scenario of test length vis-à-vis a typical need for a short CAT. ${ }^{2}$ Table 5 shows that the pattern of results for all interdimension correlations and all modes of administration was surprisingly similar to that of the longer CATs from the earlier studies; the correlations were in the same direction but had smaller overall magnitudes than did those earlier correlations.

Specifically, the regular 5-item CAT (ignoring the bidimensionality of the item pool) provided a strong correlation of one emphasized dimension's estimated theta with true theta, while the other dimension's correlation of estimated and true theta remained abysmally weak. However, the relationship between estimated theta and an average of the two dimensions' true thetas in this mode of administration was moderately strong (but less than that from the typical 20-item CAT). The 5-item contentbalanced CAT demonstrated the same pattern as did the longer content-balanced CAT: The emphasis on a single dimension at the expense of the other was somewhat muted, and the average of the two dimensions in this mode yielded a correlation between estimated and averaged true theta that was very strong. Finally, the averaged 5-item CATs yielded moderately strong correlations with both dimensions, neither dimension being accented, with a correlation between averaged true thetas and averaged 5item CATs being equal in magnitude to those correlations obtained by content-balancing. It appears on the surface that the shorter 5-item CATs might be appropriate in some testing situations, given that the emphasis is on a rapid ballpark estimation of ability.

\section{CONCLUSION}

The hypothesis that any control placed on item selection in the course of administering a CAT with multidimensional items would result in a more accurate estimation of ability than simply ignoring the nature of the 
items was supported, but not in the manner expected. If the interdimensional correlation is strong, the items behave psychometrically as if they are unidimensional anyway; controls are unwieldy and unnecessary. When lower correlations are present among items in an item pool, say, $r=0.4$ to 0.6 , content balancing is preferred to ignoring the dimensionality. Content balancing also minimized the estimation's procedure of emphasizing one of the dimensions at the expense of the other. In all cases, however, the procedure described as averaging 10-item mini-CATs appeared to best approximate the underlying true thetas and an arithmetic average of them, the target true theta.

A welcome surprise was that the shorter five-item micro-CATs behaved very much as did the standardlength CAT. This is encouraging, and it warrants further investigation into the application of CAT to competencylike tests.

Given that we have shown the various controls to be stable across two-dimensional theta space, the obvious next step would be to generalize these procedures across an $n$-dimensional theta space. For investigative purposes, we have modeled our item pools to be two-dimensional. However, it is easily conceivable that item pools-and, consequently, tests-are multidimensional to the tune of three, four, or five dimensions. Further investigation would establish the power of these item-selection controls for any such situation.

\section{REFERENCES}

ANSLEy, T. N., \& FoRsyth, R. A (1985). An examination of the characteristics of unjdimensional IRT parameter estimates derived from twodimensional data. Applied Psychological Measurement, 9, 37-48

BAKER, F. B. (1984) Ability metric transformations involved in vertical equating under item response theory Applied Psychological Measurement, 8, 261-271.
Birnanum, A. (1968). Some latent tratt models and their use in inferring an examinee's ability. In F. M. Lord \& M R. Novick (Eds.), Statistical theories of mental test scores (pp. 395-549). Reading, MA Addison-Wesley

GREAUD, V. (1987). Investigation of the unidimensionality assumption of item response theory Unpublished doctoral dissertation. Johns Hopkins University, Baltimore, MD.

GreEN, B. F (1970). Comments on tailored testing. In W Holtzman (Ed ), Computer-assisted instruction, testing, and guidance (pp. 184 197) New York. Harper \& Row

GREEN, B. F. (1983). Adaptive testing by computer In R. Ekstrom (Ed.), Measurement, technology, and individuality in education (pp. 5-12) San Francisco: Jossey-Bass.

IRONSON, G H (1983). Using item response theory to measure bias. In R. K Hambleton (Ed.), Applications of item response theon (pp. 155-174). Vancouver, BC. Educational Research Instutute of British Columbia.

LORD, F. M. (1977). Practical applications of item characteristuc curve theory. Joumal of Educational Measurement, 14, 117-138.

LORD, F. M. (1980). Applications of item response theory to practical testing problems. Hillsdale, NJ. Erlbaum.

Swaminathan, H., \& Gifford, J A (1986). Bayesian estimation in the three-parameter logistic model Psychometrika, 51, 589-601

WEISS, D. J. (1982) Improving measurement quality and efficiency with adaptive testing Applied Psychological Measurement, 6, 473-492

WINGERSKY, M. (1983) LOGIST: A program for computing maximum likelihood procedures for logistic test models In R. K. Hambleton (Ed.), Applications of item response theory (pp. 45-56). Vancouver. BC. Educational Research Institute of Britısh Colulmbia.

\section{NOTES}

1 A math word problem that requires both a component ability of analytic skills and another component ability of verbal skills would be an item demonstrating within-item multidimensionality. A test that taps two different skills or types of knowledge, such as a physics test that measures understanding of trajectories as well as refractory principles of light, is an example of between-item multudimensionality In this paper, only the latter is discussed.

2. This advantage could be generalized to drive construction of multisubject CATs, CATs that test several areas of mastery (such as a competency test). 